\title{
Thermo characteristics of steam-exploded bamboo (Phyllostachys pubescens) lignin
}

\author{
Shunliu Shao $\cdot$ Zhenfu Jin · Guifeng Wen • \\ Kenji Iiyama
}

Received: 13 August 2008/Published online: 7 May 2009

(C) The Author(s) 2009. This article is published with open access at Springerlink.com

\begin{abstract}
Bamboo (Phyllostachys pubescens) internode was subjected to steam explosion treatment to produce an excellent fiber for binderless boards. Lignin was isolated from extract-free bamboo meal with Björkman's procedure and steamexploded pulp. The self binding-mechanism was discussed by scanning electron microscopy (SEM), thermo-gravimetry (TG), differential scanning calorimetry (DSC) and analytical ozonation. It is well-known that steam explosion treatment liberates lignin from the cell wall to the fiber surface, which is the most important component relevant to binderless board production. Results of TG and DSC analyses showed that steam-exploded bamboo pulp started mass loss at lower temperature compared to bamboo internode meal. The thermal softening temperature of lignin prepared from steam-exploded pulp was much lower than that of lignin prepared from extract-free bamboo meal. This suggests that intermonomer linkages of lignin, especially $\beta$-aryl-ether linkage which is the major intermonomer linkage of lignin, were cleaved during steam explosion treatment resulting in low molecular weight phenolic compounds. The cleavage of $\beta$-aryl-ether intermonomer linkage of lignin was also confirmed by ozonation analysis.
\end{abstract}

S. Shao

Institute of Ecology, Zhejiang Forestry Academy, 310023 Hangzhou, Zhejiang, China e-mail: Shaoshunliu2000@yahoo.com.cn

\section{Z. Jin $(\bowtie) \cdot$ G. Wen}

School of Engineering, Zhejiang Forestry University, 311300 Linan, Zhejiang, China e-mail: jinzhenfu@yahoo.com.cn

K. Iiyama

Japan International Research Center for Agricultural Science, Ohwashi, Tsukuba, Ibaraki 305-8686, Japan 


\section{Introduction}

Bamboo (Phyllostachys pubescens) is an important non-wood forest resource in southeast China. It is considered as one of the important resources in wood industry to replace woody resources due to its fast growth, easy propagation, and short renovation.

Steam explosion is a physicochemical treatment of plant materials that involves an instant discharge of materials under high-pressure steam in a sealed container. The process was invented by Mason in 1926 as an alternative to chemimechanical and chemithermomechanical pulping processes (Mason 1926). Asplund (1953) applied the process to fiberboard production. He refined wood chips at elevated temperature and pressure to produce coarse and dark colored fibers for board manufacturing presenting an excellent process to produce binderless board. This was recognized not only as a very effective process to produce pulps, but also to separate plant cell wall components with low energy consumption, and it was noted as an ideal process for total utilization of lignocellulose with low emission of wastes. One of the most important findings is that the lignin portion can easily exude from the cell wall to the fiber surface during steam explosion treatment. Exuding of lignin is hardly observed in thermomechanical (TMP) and ground (GP) pulping processes (Tanahashi et al. 1983). Lignin of oil palm frond can be easily exuded from the cell wall to the fiber surface during steam explosion treatment, and binderless boards produced from steam-exploded pulp satisfied the requirement of Japan Industrial Standard (JIS A 5905 1994) (Suzuki et al. 1998; Laemsak and Okuma 2000).

The thermoplastic property of lignin is an important factor to promote the thermal adhesion of the fiberboard pulps from which the board is formed by hot pressing. Baldwin and Goring (1968) elucidated some of the practices in fiberboard technology by investigating thermoplastic and adhesive behaviors of fiberboard pulps from steam-exploded wood. They concluded that moisture of lignin and hemicelluloses not only softens but also develops adhesive properties at relatively low temperatures. Takamura (1968) also reported that the softening of lignin and hemicelluloses is markedly dependent on the water content. Sakata and Senju (1975) showed that a combined use of the plasticizer and water brought about the most effective plasticizing for the lignins. The in situ plasticization of lignin improves the final mechanical properties (Bouajila et al. 2005). Softening and steam plasticizing of lignin have been recognized for some time in various processes of the formation of board and dimensional stabilization of solid wood.

The chemical characteristics of steam-exploded bamboo pulp are reported in a previous paper (Shao et al. 2008). Lignins were isolated from extract-free bamboo internode meal, meal of binderless board prepared from steam-exploded pulp and steam-exploded pulp using Björkman's method. In this paper, thermoplastic property of steam-exploded bamboo lignin was investigated in view of binderless board production by scanning electron microscopy (SEM), thermo-gravimetry (TG) and differential scanning calorimetry (DSC). In addition, structural modification of lignin during steam-explosion was elucidated by analytical ozonation. 


\section{Experimental}

\section{Materials}

Phyllostachys pubescens Mazel ex Houzeau de Lehaie (Moso Bamboo) (5 years old) was harvested at Tanashi Experimental Station (35.7N 137.7E) of the University Forest of the University of Tokyo, Japan. Internode of bamboo was chopped to chips by a chisel (5 $\mathrm{cm}$ length and $2-3 \mathrm{~cm}$ width).

Steam explosion of chips of bamboo stem

The chips of bamboo internode (200 g) were subjected to a stem explosion digester (2 1 volume stainless steel reactor, Nittokoatsu Co. Ltd., Tsukuba, Japan). The sample was steam exploded at $2.0 \mathrm{MPa}$ and $210^{\circ} \mathrm{C}$ for $5 \mathrm{~min}$. Steam-exploded pulp was collected together with black liquor, and air-dried at room temperature.

Preparation of binderless board of steam exploded pulp

The steam exploded pulp adjusted to a moisture content of $12 \%$ by air-drying was heat-pressed by one step breathing time $(40 \mathrm{~s})$ method at $150^{\circ} \mathrm{C}$ to prepare medium density binderless boards.

\section{Lignin extraction}

Bamboo internode chip was ground by a Wiley mill to pass $420 \mu \mathrm{m}$ sieves. The ground sample was extracted with ethanol-benzene $(1: 2, \mathrm{v} / \mathrm{v})$ for $6 \mathrm{~h}$ using a Soxhlet extractor. The extract free sample was air-dried, then dried in a vacuum oven overnight at $40^{\circ} \mathrm{C}$, and finely ground with a vibratory ball mill VS-2 (Irie Shokai Co. Ltd., Tokyo, Japan) for $72 \mathrm{~h}$ with cooling by tap water flow. Dispersing solvent was not used during milling (Koshijima et al. 1972). Björkman lignin was extracted with dioxane-water $(9: 1, \mathrm{v} / \mathrm{v})$ from the finely ground sample and purified according to the procedure of Björkman (Björkman 1956), referred to as EFBL. In addition, lignin was extracted from air-dried steam-exploded pulp and purified as above (referred to as SEBL). The yields of purified lignins isolated from the finely ground sample (EFBL) and the air-dried steam-exploded pulp (SEBL) were 16.4 and $23.5 \%$ (wt\% of lignin), respectively. Lignin contents of the extract-free bamboo sample, steamexploded pulp and binderless board made by the steam-exploded pulp were determined by Klason procedure (Tappi Standard T 222om-88).

\section{Microscopy}

The steam-exploded pulp was extracted with dioxane-water $(9: 1, \mathrm{v} / \mathrm{v})$ until the color of dioxane-water solvent was clear. The original steam-exploded fibers and the fiber after extractions were observed using a SUPERSCAN SS-550 scanning electron microscope (Shimadzu, Japan) operating at $20 \mathrm{kV}$, after sputter coating with goldpalladium. 
Thermal analysis

Differential scanning calorimetry (DSC) and thermo-gravimetry (TG) analyses were performed with a Netzsch STA 409 PC Luxx (Burker, Germany) in nitrogen atmosphere $\left(25 \mathrm{~mL} \mathrm{~min}^{-1}\right)$ at a temperature range of $20-350^{\circ} \mathrm{C}$ with a heating rate of $20^{\circ} \mathrm{C} \mathrm{min}^{-1}$.

\section{Ozonation}

Ozonation analysis to discuss intermonomer linkages was carried out according to the scheme of Akiyama et al. (2002) for the extract-free and steam-exploded sample. The ozonation products were trimethylsilylated with hexamethyldisilazane and trimethylchlorosilane in dimethylsulfoxide for $30 \mathrm{~min}$ at $60^{\circ} \mathrm{C}$. The trimethylsilylated derivatives were analyzed by a Shimadzu GC-17A Gas chromatograph (Shimadzu, Japan) using NB1 capillary column $(25 \mathrm{~m} \times 0.25 \mathrm{~mm}$ id $)$ equipped with FID. Both injector and detector temperatures were $280^{\circ} \mathrm{C}$. The column temperature was kept at $120^{\circ} \mathrm{C}$ for $5 \mathrm{~min}$, and then programmed at $4^{\circ} \mathrm{C} \min ^{-1}$ to $170^{\circ} \mathrm{C}$ followed by $10^{\circ} \mathrm{C} \mathrm{min}^{-1}$ to $280^{\circ} \mathrm{C}$. Erythritol was used as an internal standard.

\section{Results and discussion}

Steam explosion is a physicochemical treatment of plant materials that involves an instant discharge of materials under high-pressure steam in a sealed container. By scanning electron microscopy it was observed that the surface of steam-exploded fibers was rough and covered with particles, while the surface of steam-exploded fibers extracted with dioxane-water $(9: 1, \mathrm{v} / \mathrm{v})$ was significantly smooth (Fig. 1a, b), suggesting that those particles are lignin liberated from cell wall by steam-explosion treatment. The suggestion was supported by the higher yield of purified lignin isolated from the air-dried steam-exploded bamboo pulp (SEBL), which was directly extracted with dioxane-water (9:1, v/v) without vibratory ball milling, compared to lignin isolated from finely ground extract-free bamboo internode sample by Björkman's procedure (EFBL). The yields of SEBL and EFBL were 23.5 and $16.4 \%$ (wt $\%$ of lignin), respectively. This result was in accordance with Suzuki et al. (1998), who reported that those materials covering the surface of pulp fiber during steam explosion were lignin, and were easily extracted with dioxane-water. It is known that the architecture of plant cell walls can be crushed by vibratory ball milling and near native lignin can be isolated by extraction with neutral solvent, which is effectively utilized for lignin structural investigations. The higher yield of lignin from SEBL than of EFBL suggests that the steam-explosion process is very effective to produce pulps and also to separate plant cell wall components with low energy consumption. Tanahashi et al. (1983) concluded that the brown colored oily substances detected both inside and outside of exploded wood pulp fibers were lignin, originating from both middle lamellae and secondary walls liberated from cell wall polysaccharides during steam explosion. Production of these substances is 


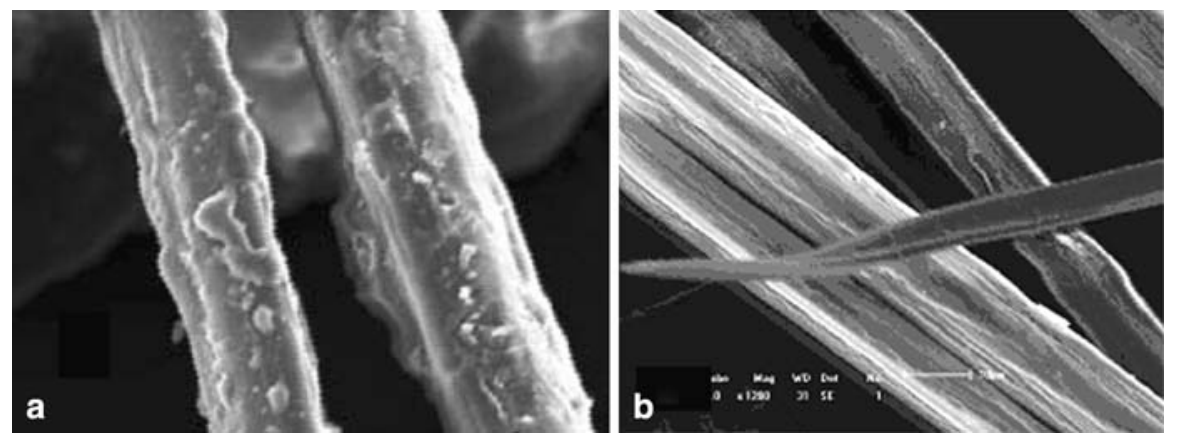

Fig. 1 Scanning electron micrograms of (a) steam-exploded pulp of bamboo and (b) steam-exploded pulp extracted with dioxane-water $(9: 1, \mathrm{v} / \mathrm{v})$
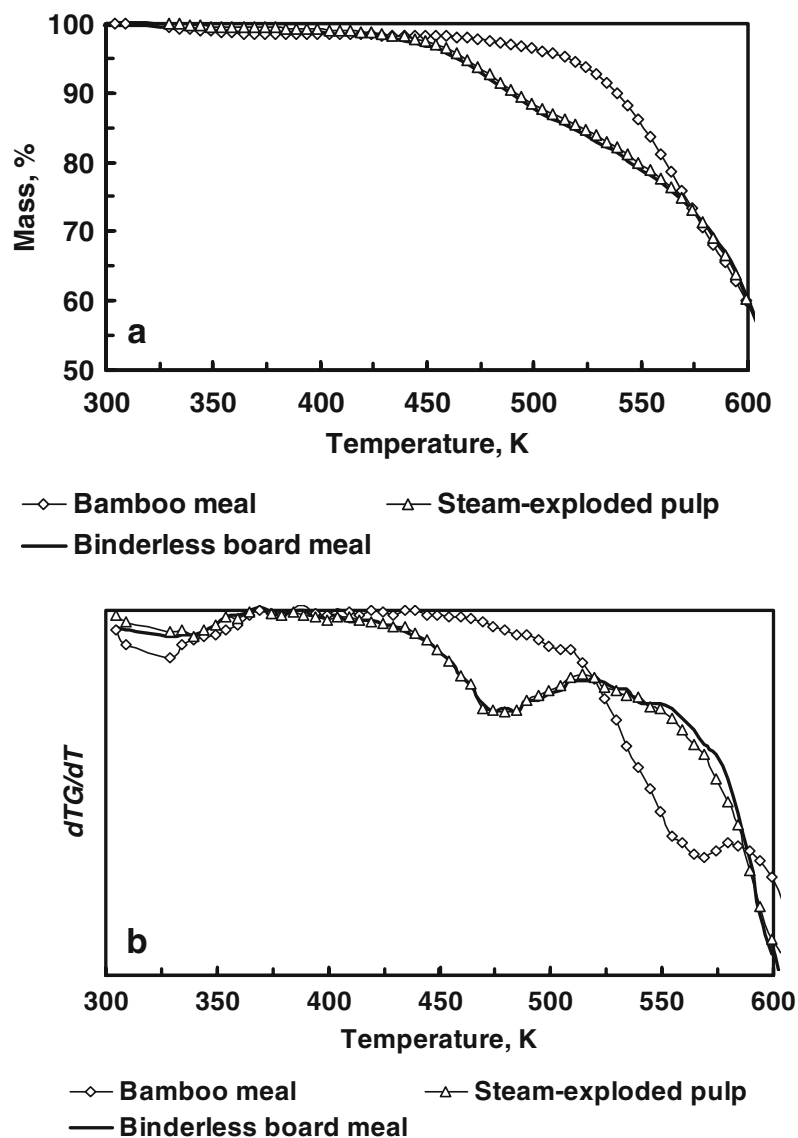

Fig. 2 TG curves (a) and their differential curves (b) of extract-free bamboo meal, steam-exploded pulp of bamboo and binderless board meal prepared by steam-exploded pulp of bamboo 

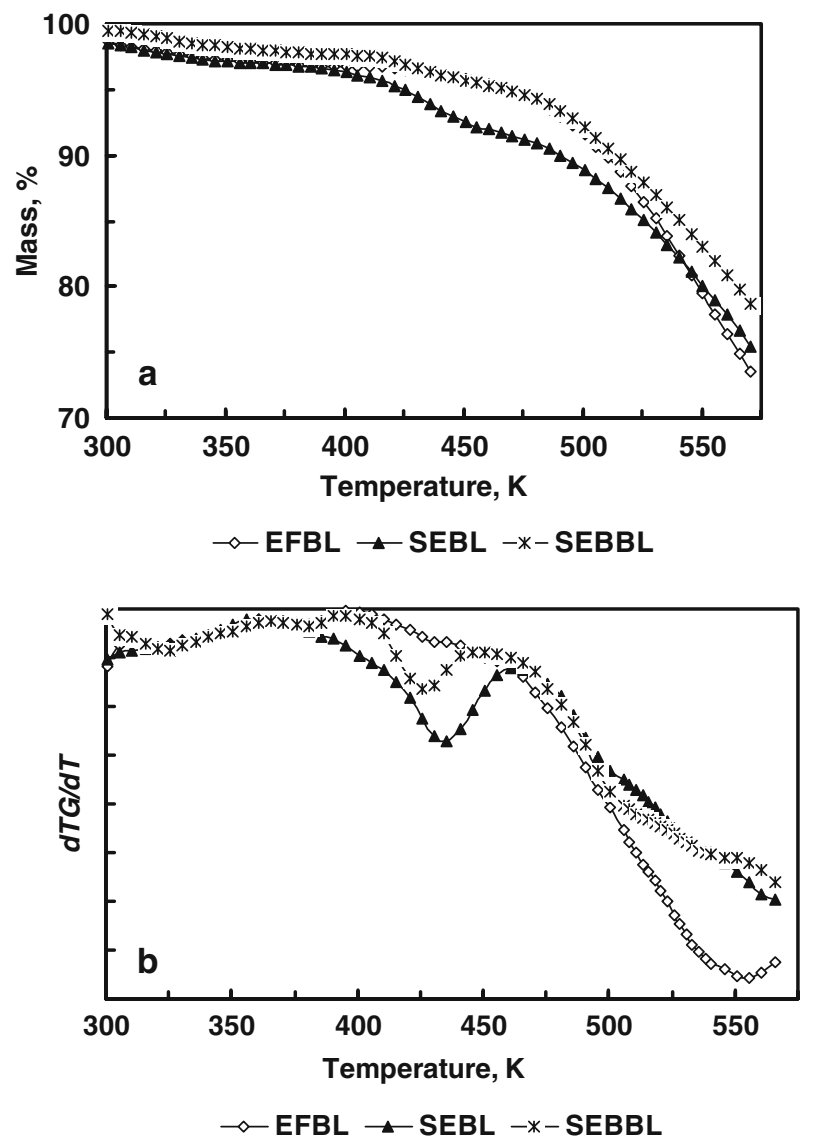

Fig. 3 TG curves (a) and their differential curves (b) of isolated lignins from extract-free bamboo (EFBL), steam-exploded pulp of bamboo (SEBL) and binderless board made by steam-exploded pulp (SEBBL)

expected as one of the important characteristics of the steam explosion process, because those phenomena were hardly detected in thermomechanical pulp (TMP) and ground pulping (GP) processes.

Thermal weight loss of the extract-free bamboo internode meal started at about $473 \mathrm{~K}$ and it occurred in one step. Both the steam-exploded pulp and the meal of binderless board made by steam-exploded pulp started sharp weight loss at $437 \mathrm{~K}$ and the curve characterized two steps (Fig. 2a, b). The rate of weight loss reached a maximum at $573 \mathrm{~K}$ and weight loss was almost $25 \%$ at the temperature. Such weight loss is mainly due to the thermal decomposition involving discharge of $\mathrm{CO}_{2}$, $\mathrm{CO}$, and $\mathrm{H}_{2} \mathrm{O}$ (Jiang et al. 2007). Lignins from EFBL and SEBL started thermal weight losses at 403 and $383 \mathrm{~K}$ (Fig. 3a, b), which were lower than that of the extract-free bamboo internode meal and steam-exploded bamboo pulp (Fig. 2a, b), respectively. Thermal weight losses within the temperature range of 383-513 K were 7.5 and $8.9 \%$ (based on lignin) for EFBL and SEBL, respectively. 


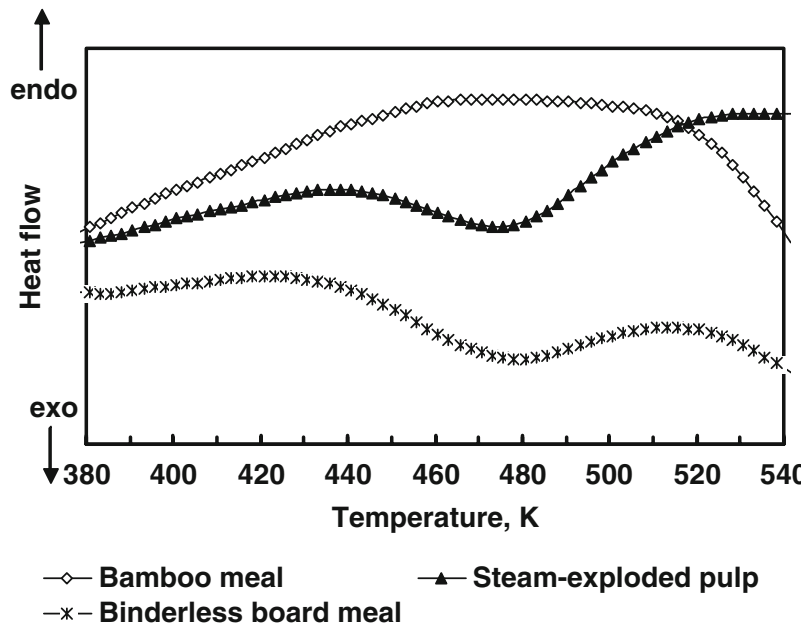

Fig. 4 DSC curves of extract-free bamboo meal, steam-exploded pulp of bamboo and binderless board meal prepared by steam-exploded pulp of bamboo

Lignin is a thermoplastic amorphous polymer, i.e., lignin undergoes what is known as a glass transition. When lignin softens, it becomes tacky and exhibits auto-adhesion, and the significance of lignin softening in the production of fiberboard is expected to be particularly important (Goring 1971). The glass transition temperature of bamboo powder is at $538 \mathrm{~K}$, higher than that for cellulose at about $503 \mathrm{~K}$ ( $\mathrm{Li}$ et al. 2006). Dry cellulose softens at 503-523 K because of its crystalline nature, and this softening temperature did not fall a lot by the presence of moisture. Hemicelluloses and lignin soften at 433-473 K, but unlike cellulose, the softening temperatures of these amorphous components undergo a marked fall with moisture content (Baldwin and Goring 1968). Both the steam-exploded pulp and the steam-exploded binderless board meal presented the glass transition temperature around $448 \mathrm{~K}$ (Fig. 4). The glass transition temperatures of hemicelluloses and lignin were markedly lowered after steam-explosion treatment. The low glass transition temperature of the steam-exploded pulp indicates cellulose, hemicelluloses and lignin being separated by steam-explosion treatment. The glass transition temperature of lignin ranges from 400 to $466 \mathrm{~K}$ depending on their source and method of isolation (Goring 1971). The SEBL showed glass transition temperature at $409 \mathrm{~K}$, which was much lower than that of the bamboo internode meal at about $435 \mathrm{~K}$, suggesting that lignin was depolymerized during steam-explosion treatment. It is well known that glass transition temperature of dioxane lignin drops with the decrease of its molecular weight (Goring 1971). The glass transition temperature of SEBL was lower than the board press temperature at $423 \mathrm{~K}$, which is expected to contribute to the adhesive behavior of the fiberboard.

On the DSC curves, EFBL and SEBL showed the maximum endothermic peaks at 442 and $429 \mathrm{~K}$, and the endothermic energies were 10.6 and $84.7 \mathrm{~J} \mathrm{~g}^{-1}$, respectively (Fig. 5). The lower maximum endothermic peak and the higher endothermic energy of SEBL indicate that the lower molecular weight of SEBL 


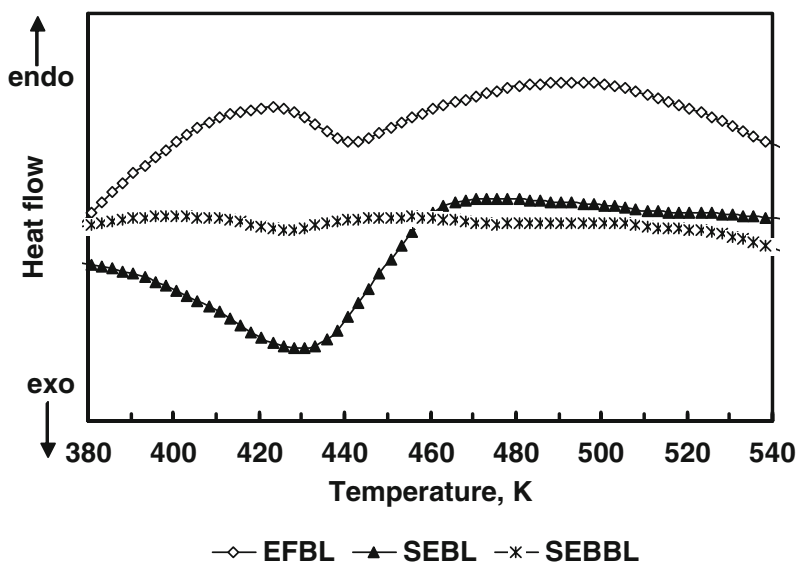

Fig. 5 DSC curves of isolated lignins from extract-free bamboo (EFBL), steam-exploded pulp of bamboo (SEBL) and binderless board made by steam-exploded pulp (SEBBL)

compares to EFBL due to the cleavage of ester bond between lignin and $p$-coumaric acid during steam explosion treatment in addition to the cleavage of $\beta$-aryl-ether intermonomer linkage ( $\beta$-O-4 linkage) of lignin. This suggestion was supported by the results of the lower $p$-hydroxybenzaldehyde in products of alkaline nitrobenzene oxidation and higher value of phenolic hydroxyl group of the steam-exploded pulp, which indicates that $p$-coumaric acid esterified to lignin side chain was cleaved by steam-explosion treatment and release of free phenolic hydroxyl groups (Shao et al. 2008).

The cleavage of $\beta$-aryl-ether intermonomer linkage ( $\beta$-O-4 linkage) of lignin was confirmed by ozonation analysis. Marchessault et al. (1982) reported that $\beta-O-4$ linkage was significantly cleaved during steam-explosion process. The $\beta-O-4$ linkage is a major intermonomer linkage in lignin, and the erythro form of the $\beta$-O-4 linkages predominates in angiosperms (Akiyama et al. 2005; Matsumoto et al. 1993). By analytical ozonation, erythronic and threonic acids are obtained from erythro and threo forms of $\beta-O-4$ structure, respectively (Akiyama et al. 2005; Matsumoto et al. 1993). The total yields of erythronic and threonic acids range from 220 to $370 \mathrm{mmol}$ $\left(200 \mathrm{~g} \mathrm{lignin}^{-1}\right.$ for various woody angiosperm lignins (Akiyama et al. 2005).

Table 1 Analytical ozonation products of EFBL and SEBL (mmol $\left.(200 \mathrm{~g} \mathrm{lignin})^{-1}\right)$

\begin{tabular}{llc}
\hline & EFBL & SEBL \\
\hline $\mathrm{E}$ & 71 & 56 \\
$\mathrm{~T}$ & 50 & 43 \\
Total & 121 & 99 \\
$\mathrm{E} /(\mathrm{E}+\mathrm{T})$ & 0.59 & 0.57 \\
\hline
\end{tabular}

EFBL: Lignin from extract-free bamboo internode with Björkman's procedure

SEBL: Lignin from steam-exploded bamboo pulp extracted with $90 \%$ dioxane (v/v) and purified with Björkman's procedure

$E$ erythronic acid, $T$ threonic acid 
The total yield of erythronic and threonic acid in ozonation products of EFBL was $121 \mathrm{mmol}$ (200 $\mathrm{g}$ lignin $)^{-1}$ which was higher than the values of SEBL (Table 1). This result clearly suggested significant cleavage of $\beta-O-4$ intermonomer linkages during steam explosion treatment. The cleavage of $\beta$-aryl-ether intermonomer linkages was also confirmed by ${ }^{1} \mathrm{H}$ and ${ }^{13} \mathrm{C}$ NMR spectroscopy (Suzuki et al. 1998; Shao et al. 2008).

\section{Conclusion}

1. Lignin liberated from cell wall by steam-explosion treatment accumulated on the surface of the steam exploded pulp to give surface roughness.

2. The glass transition temperature of SEBL was at $409 \mathrm{~K}$, which was significantly lower than that of EFBL, suggesting that lignins were depolymerized during steam-explosion treatment. They are expected to contribute to the adhesive behavior for the formation of binderless boards.

3. The intermonomer linkage of lignin and ester bond between lignin and $p$-coumaric acid was cleaved by steam-explosion treatment resulting in low molecular weight lignin rich in phenolic hydroxyl end groups.

Acknowledgments This work was completed with the financial support of the National Natural Science Fundation of China (30671636), and Agricultural Key Research Project of Zhejiang Province of China (2006C22077). The authors take pleasure in acknowledging the skillful assistance of Ms. Wenzhu Li and Fengzhu Lu in operating TG/DSC analyses, and SEM observation.

Open Access This article is distributed under the terms of the Creative Commons Attribution Noncommercial License which permits any noncommercial use, distribution, and reproduction in any medium, provided the original author(s) and source are credited.

\section{References}

Akiyama T, Sugimoto T, Matsumoto Y, Meshitsuka G (2002) Erythro/threo ratio of $\beta$ - $O-4$ structures as an important structural characteristic of lignin. Part 1. Improvement of ozonation method for the quantitative analysis of lignin side-chain structure. J Wood Sci 48:210-215

Akiyama T, Goto H, Nawawi DS, Syafii W, Matsumoto Y, Meshitsuka G (2005) Erythro/threo ratio of $\beta-O-4$ structures as an important structural characteristic of lignin. Part 4: variation in the erythro/ threo ratio in softwood and hardwood lignins and its relation to syringyl/guaiacyl ratio. Holzforschung 59:276-281

Asplund A (1953) The origin and development of the defibrator process. Sven Papperstidn 56:550-558

Baldwin SH, Goring DAI (1968) The thermoplastic and adhesive behaviour of thermomechanical pulp from steamed wood. Sven Papperstidn 71:646-650

Björkman A (1956) Studies on finely divided wood. Part 1. Extraction of lignin with neutral solvents. Sven Papperstidn 59:477-485

Bouajila J, Limare A, Joly C, Dole P (2005) Lignin plasticization to improve binderless fiberboard mechanical properties. Polym Eng Sci 80:9-816

Goring DAI (1971) Polymer properties of lignin and lignin derivatives. In: Sarkanen KV, Ludwig CH (eds) Lignins. Wiley, New York, pp 659-768

Jiang Z, Zhang D, Fei B, Yue Y, Chen X (2007) Thermal characteristics of bamboo. http://info. ewood.cn/edu/bamboo 
JIS A 5905 (1994) Fibreboards. Japan Industrial Standard

Koshijima T, Taniguchi T, Tanaka R (1972) Lignin-carbohydrate complex. I. Influences of milling of wood upon the Björkman LCC. Holzforschung 26:211-217

Laemsak N, Okuma M (2000) Development of boards made from oil palm frond II: properties of binderless boards from steam-exploded fibers of oil palm frond. J Wood Sci 46:322-326

Li WZ, He YJ, Chen P (2006) The factors influencing the characteristics of bamboo pyrolysis, a hot analytic approach. J Bamboo Res 25(2):31-34

Marchessault RH, Coulombe S, Morikawa H, Robert D (1982) Characterization of aspen exploded wood lignin. Can J Chem 60(18):2372-2382

Mason WH (1926) Process and apparatus for disintegration of wood and the like. US Patent No 1578609

Matsumoto Y, Minami K, Ishizu A (1993) Structural study on lignin by ozonation. The erythro and threo ratio of the $\beta-O-4$ structure indicates how lignin polymerizes. Mokuzai Gakkaishi 39:734-736

Sakata I, Senju R (1975) Thermoplastic behavior of lignin with various synthetic plasticizers. J Appl Polym Sci 19:2799-2810

Shao S, Wen G, Jin Z (2008) Changes in chemical characteristics of bamboo (Phyllostachys pubescens) components during steam explosion. Wood Sci Technol 42:439-451

Suzuki S, Shintani H, Park SK, Saito K, Lemsak N, Okuma M, Iiyama K (1998) Preparation of binderless boards from steam-exploded pulps of oil palm (Elaeis guneenisis Jaxq.) fronds and structural characteristics of lignin and wall polysaccharides in stem exploded pulps to be discussed for selfbindings. Holzforschung 52:417-426

Takamura N (1968) Studies on hot pressing and drying process in the production of fibreboard. III. Softening of fibre components in hot pressing of fibre mat. Mokuzai Gakkaishi 14(2):75-79

Tanahashi M, Takada S, Aoki T, Goto T, Higuchi T, Hanai S (1983) Characterization of explosion wood. 1. Structure and physical properties. Wood Res 69:36-51 